\title{
NONLINEAR GENERALIZED MINIMUM VARIANCE CONTROL UNDER ACTUATOR SATURATION ${ }^{1}$
}

\author{
Michael J. Grimble* Pawel Majecki* \\ * Industrial Control Centre, University of Strathclyde, \\ Glasgow, UK \\ p.majecki@strath.ac.uk
}

\begin{abstract}
A new Generalized Minimum Variance control law has been derived recently for the control of nonlinear multivariable systems. In this paper we restrict our interest to single-input, single-output plants with input nonlinearities in the form of hard actuator limits. Since in real systems saturation always exists in some form, e.g. as a result of valve opening limits or finite power supply, this is a natural case to consider. One of the well-known problems associated with input saturation is the integral windup phenomenon, which occurs whenever the controller includes integral action. In this paper, we show that the classical form of the "anti-windup" mechanism can be obtained within the Nonlinear GMV controller framework by a suitable selection of the design parameters. The advantage of the approach is that the anti-windup mechanism is obtained naturally from the optimization problem. There is also the possibility that the technique can be extended for other specialized nonlinear compensation problems. Copyright 2005 IFAC
\end{abstract}

Keywords: Actuator saturation, Nonlinear control, Stochastic control

\section{INTRODUCTION}

The subject of actuator saturation has received considerable attention in the literature on control systems design and tuning. This is mainly due to the fact that certain limits on the control action are always present in real systems, caused usually by a finite energy supply or physical constraints of the actuation element. It is well known that saturation, if its effects are ignored in the design, may lead to some significant deterioration in the control performance, and in some cases it may even destabilize the closed-loop system (Seron et al., 1994; Levine, 1996; Goodwin et al., 1993).

\footnotetext{
1 We are grateful for the support of the Engineering and Physical Sciences Research Council on the Platform Grant Project No GR/R04683/01
}

The main problem with saturation occurs when the control algorithm includes integral action. When the saturation constraint becomes active, the system effectively operates in open loop, with the process manipulated variable fixed at one of its limits. No further control action can then be produced to reduce the control error. The integrator however is ignorant of this and causes the controller output to increase, resulting in undesirable transients when the system eventually gets back to the working range. This phenomenon is known as integral wind-up. The actuator saturation imposes a hard constraint on the controller performance and cannot be fully compensated the best that can be done is to keep the control action within or on the saturation limits and to make sure that the integral component of the controller does not increment its internal state un- 
der saturation conditions. The special controller structures achieving these objectives are generally known as "anti-windup mechanisms", and many different approaches to this problem have been developed (Goodwin, 1972; Åström, 1984; Hanus et al., 1987).

In principle, the anti-windup mechanism is not an integral part of any particular control law and can always be used as a separate compensator of special type, when the saturation is present and the controller includes integral action (as is the case in many practical applications). It can basically be considered a heuristic modification of the existing controller structure. In this paper, we discuss such a scheme in the context of the Nonlinear GMV control (Grimble, 2004; Majecki and Grimble, 2004) and show that the "classical" anti-windup algorithm results from an optimization problem by a suitable choice of the design parameters.

The paper is organized as follows: in section 2, the system description and the NGMV cost function are introduced; in Section 3, the optimal control solution is presented, and the possible application to controller benchmarking is mentioned; Section 4 deals with the main topic, which is the actuator saturation; it describes a modification to the cost criterion and illustrates the results with a simulated example; the paper closes with conclusions in Section 5.

\section{STOCHASTIC SYSTEM DESCRIPTION AND PERFORMANCE CRITERION}

The system shown in Fig. 1 is of restricted generality and is carefully chosen so that simple results are obtained. The plant itself is nonlinear and may have quite a general form which might involve state-space, transfer operators, neural networks or even nonlinear function look-up tables. However, the reference and disturbance signals are assumed to have linear time-invariant model representations. This is not very restrictive, since in many applications the models for the disturbance and reference signals are only LTI approximations.

A nonlinear plant model can be written in the following form:

$$
(\mathcal{W} u)(t)=z^{-k}\left(\mathcal{W}_{k} u\right)(t)
$$

where $k$ denotes the magnitude of the plant timedelay.

\subsection{Signals}

The signals shown in the system model of Fig. 1 may be listed as follows:

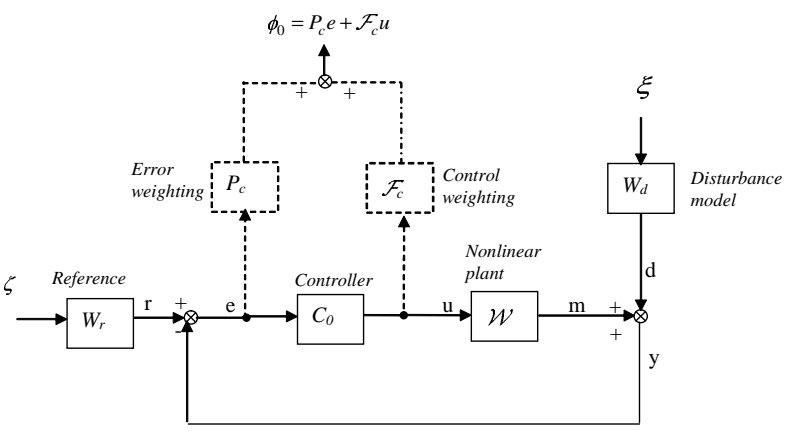

Fig. 1. Single Degree of Freedom Feedback Control System for the Nonlinear Plant (inferred output $\phi_{0}$ is dependent on the weightings shown dotted)

Error signal:

$$
e(t)=r(t)-y(t)
$$

\section{Plant output:}

$$
y(t)=(\mathcal{W} u)(t)+d(t)
$$

\section{Reference signal:}

$$
r(t)=W_{r} \zeta(t)
$$

\section{Disturbance signal:}

$$
d(t)=W_{d} \xi(t)
$$

The power spectrum for the combined reference and disturbance signal $f=r-d$ can be computed as:

$$
Y_{f f}=\Phi_{r r}+\Phi_{d d}=W_{r} W_{r}^{*}+W_{d} W_{d}^{*}
$$

and the strictly minimum phase generalized spectralfactor $Y_{f}$ may be computed using:

$$
Y_{f} Y_{f}^{*}=\Phi_{f f}
$$

Note that a measurement noise model has not been included to simplify the equations. This is appropriate so long as the control cost-function weighting, introduced in the next section, ensures the controller rolls-off at high frequencies.

\subsection{NGMV cost function}

The optimal NGMV control problem involves the minimization of the variance of the signal $\phi_{0}(t)$ in Fig. 1. This signal involves a dynamic cost function weighting $P_{c}\left(z^{-1}\right)$ on the error signal, represented in transfer-function form as: $P_{c}=$ $\frac{P_{c n}}{P_{c d}}$. It also includes a nonlinear dynamic control signal costing operator term: $\left(\mathcal{F}_{c} u\right)(t)$. The choice of the dynamic weightings is critical to the design 
and typically $P_{c}$ is low-pass and $\mathcal{F}_{c}$ is constant or a high-pass transfer. The signal:

$$
\phi_{0}(t)=P_{c} e(t)+\left(\mathcal{F}_{c} u\right)(t)
$$

is to be minimized in a variance sense, so that the cost index to be minimized:

$$
J=E\left\{\phi_{0}^{2}(t)\right\} .
$$

If the plant time-delay is of magnitude $k$, this implies the control at time $t$ affects the output $k$ steps later. For this reason the control weighting can be defined as:

$$
\left(\mathcal{F}_{c} u\right)(t)=z^{-k}\left(\mathcal{F}_{c k} u\right)(t)
$$

Typically this will be a linear operator but it may also be chosen to be nonlinear to compensate for the plant input nonlinearities in appropriate cases, as discussed in Section 4. The delay-free control weighting operator $\mathcal{F}_{c k}$ is assumed to be invertible.

\subsection{Generalized plant}

One approach to the above control problem is to reformulate the NGMV criterion as a simpler nonlinear minimum variance problem for a generalized plant. The expression for the controller error can be written as:

$$
e(t)=-z^{-k}\left(\mathcal{W}_{k} u\right)(t)+Y_{f} \varepsilon(t)
$$

where $\varepsilon(t)$ is a zero-mean, unity-variance white noise process and $Y_{f}$ represents the combined effect of all the stochastic inputs to the system.

The generalized output $\phi_{0}(t)$ can be rewritten as follows:

$$
\begin{gathered}
\phi_{0}(t)=P_{c}\left(-z^{-k}\left(\mathcal{W}_{k} u\right)(t)+Y_{f} \varepsilon(t)\right)+\left(\mathcal{F}_{c} u\right)(t) \\
=z^{-k}\left(\mathcal{F}_{c k}-P_{c} \mathcal{W}_{k}\right) u(t)+P_{c} Y_{f} \varepsilon(t)
\end{gathered}
$$

Notice that the non-linear operator in this last expression can be considered a "generalized plant" and the notation implies that:

$$
\left(P_{c} \mathcal{W}-\mathcal{F}_{c}\right) u=P_{c}(\mathcal{W} u)(t)-\left(\mathcal{F}_{c} u\right)(t)
$$

\section{OPTIMAL NONLINEAR GMV PROBLEM AND SOLUTION}

The key step in the derivation of the NGMV control law is to split the cost function (9) into two statistically independent terms, one of which is independent, and the other dependent on the controller. The control law then results by simply setting the latter to zero.
Dividing the weighted disturbance term into unpredictable and predictable components using the Diophantine identity $\left(\operatorname{deg} F_{0}<k\right)$ :

$$
P_{c} Y_{f}=F_{0}+z^{-k} R,
$$

equation (12) can be rewritten as:

$\phi_{0}(t)=F_{0} \varepsilon(t)+z^{-k}\left[\left(\mathcal{F}_{c k}-P_{c} \mathcal{W}_{k}\right) u(t)+R \varepsilon(t)\right]$

To compute the optimal control signal, inspect the form of equation (15). Since the degree of the polynomial $F_{0}$ is required to be less than $k$, it follows that the first and the remaining terms are statistically independent, even though the second term involves a nonlinear operator.

Furthermore, the first term on the right of (15) is independent of the control action and the smallest variance is achieved when the remaining terms are set to zero. The optimal control signal must therefore satisfy:

$$
u^{o p t}(t)=-\left(\mathcal{F}_{c k}-P_{c} \mathcal{W}_{k}\right)^{-1} R \varepsilon(t)
$$

which, after some algebraic manipulations, can be rewritten as

$$
u^{o p t}(t)=-\left[\left(\mathcal{F}_{c k}-F_{0} Y_{f}^{-1} \mathcal{W}_{k}\right)^{-1} R Y_{f}^{-1} e\right](t)
$$

The above result indicates that the restriction on the choice of the cost weightings is that the operator $\left(P_{c} \mathcal{W}_{k}-\mathcal{F}_{c k}\right)$ must have a stable inverse for the relevant input signal range.

The general NGMV controller structure, based on equation (17), is shown in Fig. 2. Since the controller inner loop contains the delay-free model of the plant, an algebraic loop may arise in the actual implementation. This problem can be avoided by extracting that part of the nonlinear operator that depends upon the present value of the control action and appropriately modifying the controller structure. Alternatively, the algebraic loop may be solved using iterative methods, or an additional delay may be introduced into the loop.

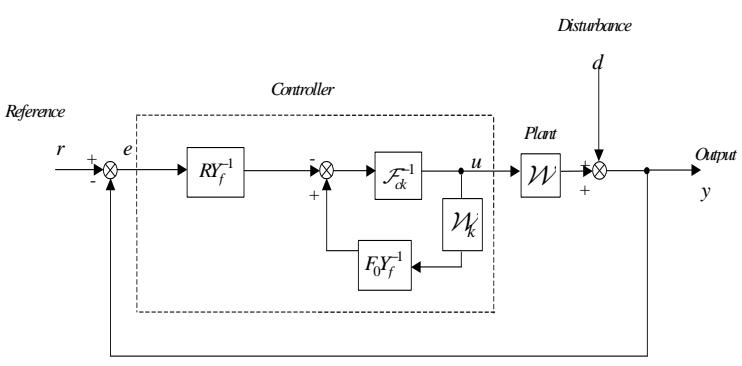

Fig. 2. NGMV controller structure

\subsection{Application to controller performance assessment}

The generalized output under NGMV optimal control is a linear moving-average time series: 


$$
\phi_{0}^{o p t}(t)=F_{0} \varepsilon(t) .
$$

Its variance (the minimum value of the cost function) follows as:

$$
J_{\min }=\operatorname{Var}\left[F_{0} \varepsilon(t)\right]=\sum_{i=0}^{k-1} f_{i}^{2}
$$

and depends only on the combined disturbance and reference model, and the plant time delay.

This theoretically achievable lower bound on the value of the cost function can be estimated from routine closed-loop operating data. The numerical algorithms that can be used for this purpose have been described in (Desborough and Harris, 1992; Huang and Shah, 1999), and their application to NGMV benchmarking has been discussed in (Majecki and Grimble, 2004). This provides a means of assessing the performance of nonlinear control systems.

\section{THE CASE OF ACTUATOR SATURATION}

Actuator saturation is a common cause of poor control performance and may even lead to instability of a control system. These detrimental effects are particularly severe when the controller includes integral action (as in most cases). The integrator increases without constraints under a constant error signal and needs time to get back to the working range when the error reverses. This usually results in some undesirable transient behaviour. Whilst it is not possible to fully compensate for the decrease in gain caused by the saturation, there are standard methods of avoiding the phenomenon of the so-called integral wind-up.

A number of heuristic methods have been developed to deal with this problem, and one of such "anti-windup" mechanisms is presented in Fig. 3, for the simple case of PI controller with hard actuator saturation. The idea is to calculate the difference between the output and input of the actuator (or its model, if the actual output is not available) and feed it back to the input of the controller integrator. The speed of response can be set by the parameter $T_{t}$. In this section we will show that a similar mechanism can be obtained within the NGMV framework by a special choice of the control weighting. Thus the heuristic structure shown in Fig. 3 will attain a more solid justification, as a solution to a formal optimization problem.

\subsection{NGMV solution of the anti-windup problem}

Let the function $\operatorname{Sat}(\cdot)$ denote a saturation type characteristic, which might be a part of the phys-

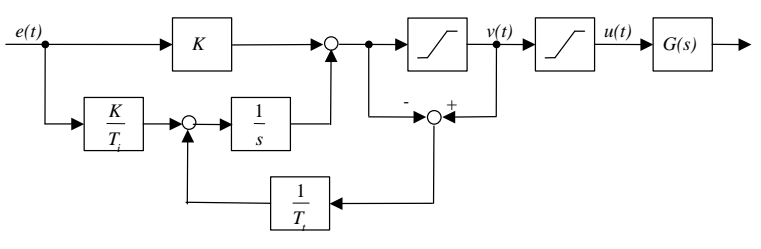

Fig. 3. "Classical" anti-windup mechanism for a continuous-time PI controller

ical plant input subsystem. However, the system may also have hard constraints on the input signals which can be represented by this function.

For simplicity let the gain in the linear region of this function be taken as unity. Denote a nominal invertible choice of the control weighting as $\hat{\mathcal{F}}_{c}=$ $z^{-k} \hat{\mathcal{F}}_{c k}$ and consider the following modification:

$\left(\mathcal{F}_{c k} u\right)(t)=\left(\hat{\mathcal{F}}_{c k} u\right)(t)+\frac{\rho}{1-z^{-1}}\left[u(t)-\operatorname{Sat}\left(u_{t}\right)\right](20)$

where $\rho$ is a positive scalar weighting. The second term in (20) does not affect the overall weighted control signal in the "linear" region but becomes active under saturation conditions. The rationale behind such a choice of the weighting is to penalize the excessive control action and therefore prevent the integral wind-up.

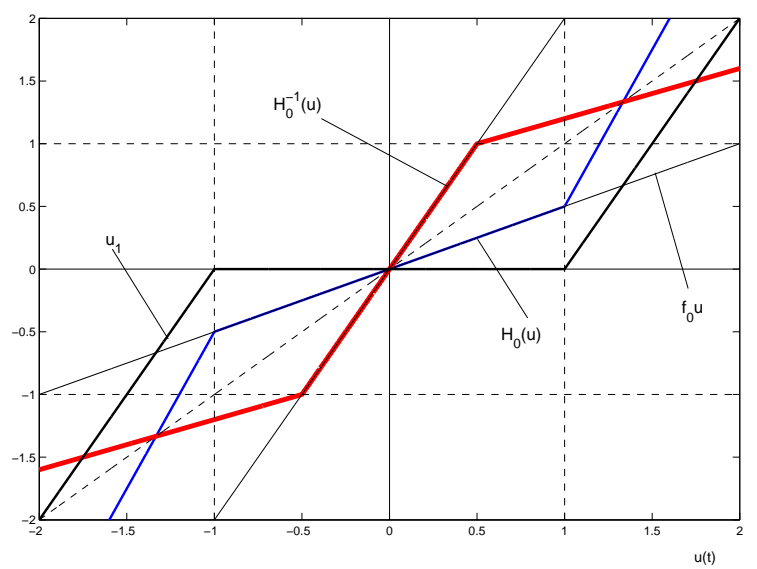

Fig. 4. Invertibility of the static function $H_{0}(u(t))$

That the modified control weighting operator (20) is also invertible, can be seen from the following argument. Assume for simplicity that the nominal control weighting is linear: $\hat{\mathcal{F}}_{c k}=\hat{F}_{c k}$ and write its impulse response as $\hat{F}_{c k}=f_{0}+z^{-1} \hat{F}_{c k 1}$. Similarly, the first "impulse response coefficient" of the second (nonlinear) term in (20) can be found as $\rho u_{1}(t)$ where $u_{1}(t)=\left[u(t)-\operatorname{Sat}\left(u_{t}\right)\right]$ represents the deadzone operator. Collecting together the terms related to the current value of $u(t)$, the equation (20) can be written as

$\left(\mathcal{F}_{c k} u\right)(t)=H_{0}(u(t))+\hat{F}_{c k 1} u(t-1)+\frac{\rho}{1-z^{-1}} u_{1}(t-1)$

where $H_{0}(u(t))=f_{0} u(t)+\rho\left[u(t)-\operatorname{Sat}\left(u_{t}\right)\right]$ is a static function of the current value of $\mathrm{u}(\mathrm{t})$ 
and is invertible, as illustrated in Fig. 4. In this figure, the two components of $H_{0}(\cdot)$ have been drawn separately, as well as their sum and its inverse (in this particular example $0<f_{0}<1$ ). The overall control weighting has therefore been shown to be invertible, and this is also true for a nonlinear nominal control weighting, as long as the invertibility assumption is satisfied. In that case, however, $f_{0}$ in general will not be a constant but rather a static function of $u(t)$.

Note that the above argument fails if $\hat{\mathcal{F}}_{c k}$ is not invertible, e.g. equals zero. The "static" part of the control weighting then represents a pure deadzone operator, which is not strictly monotonic and therefore not invertible. If it were, that would correspond to the ideal compensation of the actuator saturation, which is of course impossible. The best that can be done is to reduce the detrimental effects of the saturation, and this is done in our case by an appropriate modification of the actual nonlinearity.

The optimal control action (17) can now be rewritten as

$$
\begin{aligned}
u(t) & =\hat{\mathcal{F}}_{c k}^{-1}\left[F_{0} Y_{f}^{-1}\left(\mathcal{W}_{k} u\right)(t)-\right. \\
& \left.\frac{\rho}{1-z^{-1}} u_{1}(t)-R Y_{f}^{-1} e(t)\right]
\end{aligned}
$$

Fig. 5 shows the new arrangement, where $\mathcal{W}_{k}$ is the delay-free model of the plant. Note that the error weighting $P_{c}$ is assumed to include an integrator to penalize non-zero steady-state errors, and that both integrators have been moved inside the loop to avoid the state mismatch (to account for this, $R_{0}=\left(1-z^{-1}\right) R$ in the diagram). The "anti-windup" feedback is only active under saturation and prevents the integrator state from increasing. The speed of response is determined by parameter $\rho$. In the linear region the control law collapses to the nominal case. By comparing Figs. 3 and 5 it may be concluded that the optimal control action obtained by introducing the modified control weighting (20) has the same structure as the classical anti-windup scheme.

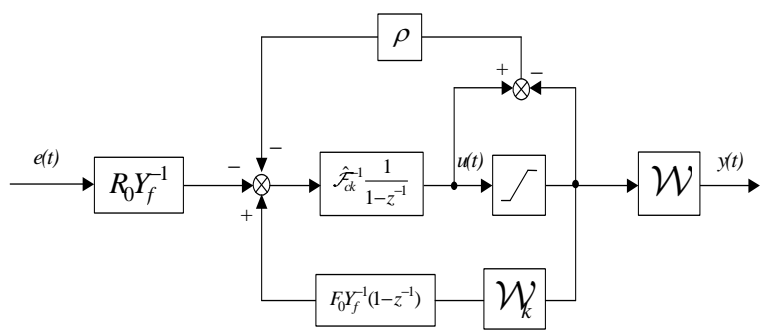

Fig. 5. Control signal generation and controller modules for the NGMV + Anti-Windup
4.2 Example: NGMV controller design for a linear plant with input saturation

There are two main points to illustrate with this example:

- compare the performance of the NGMV controller for the nominal and modified control weighting

- investigate the influence of the parameter $\rho$ in the anti-windup loop.

The actual plant is linear, and is modelled by a simple first-order lag in series with an integrator:

$$
W(s)=\frac{K}{s(\tau s+1)}
$$

with the nominal settings $\tau=0.5 s$ and $K=1$. This setup may represent the dynamics of a DC motor, where the input is the voltage applied to the motor, and the output is the shaft position. Obviously, there will always be a maximum permissible voltage that can be applied, and this constraint can be approximately modelled using the nonlinear saturation element. The saturation function for this example is symmetric and constrains the actual input to the plant to the range $[-a, a]$, with the nominal setting $a=1$. Since the plant includes an integrator, arbitrary setpoints may in principle be achievable, however the transient performance may be deteriorated due to the saturation and integral wind-up. The modification of the control weighting will be used to tackle this problem.

A nonlinear GMV controller design has been performed for this system, resulting in the following weighting selection:

$$
P_{c}=\frac{1-0.8 z^{-1}}{1-z^{-1}}, \quad \hat{\mathcal{F}}_{c k}=-0.2
$$

The final design includes also the nonlinear modification as in equation (20). The controller includes the discretized model of the plant, with the sampling period $T_{s}=0.1 s$.

Fig. 6 shows the simulation results for the stepchange reference signal and the values of parameter $\rho$ ranging from $10^{-4}$ to 50 . The horizontal dash-dotted lines in the control signal plot correspond to saturation limits, and in both plots the direction of increasing $\rho$ has been indicated. The value $\rho=10^{-4}$ is close to the 'no anti-windup' case, and the larger $\rho$ becomes, the better the correcting action that prevents the state of the integrator from increasing.

\section{CONCLUSION}

Nonlinear GMV controller design in the presence of hard actuator constraints has been considered. It was shown that by a particular choice of the 

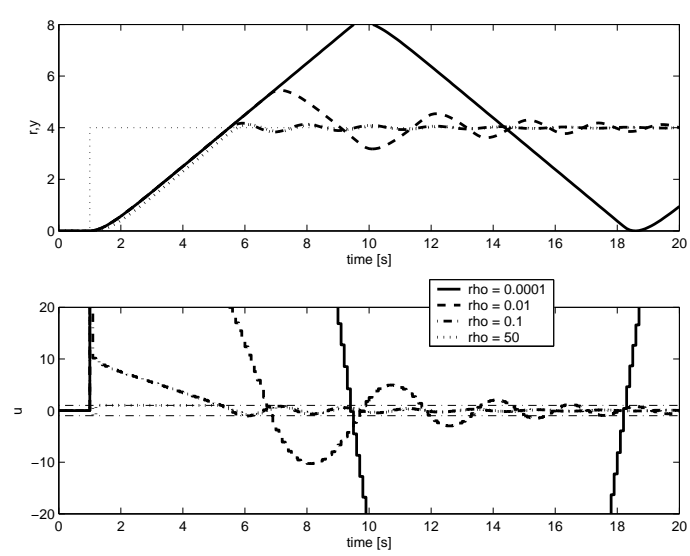

Fig. 6. Simulation results for different values of the parameter $\rho(\rho=0.0001,0.01,0.1,50)$

weighting transfer functions it is possible to obtain a control structure that is equivalent to the wellknown anti-windup scheme. The approach may be extended for other types of nonlinear problems (e.g. deadzone). That is, the cost index can have additional nonlinear terms to modify the controller characteristics in a way that is impossible in linear optimal control design.

\section{REFERENCES}

Åström, K.J. (1984). Computer Controlled Systems. 2nd ed.. Prentice Hall, Englewood Cliffs. NJ.

Desborough, L. and T. Harris (1992). Performance assessment measures for univariate feedback control. The Canadian Journal of Chemical Engineering Vol.70, pp.1186-1197.

Goodwin, G.C. (1972). Amplitude-constrained minimum variance controller. Electronics Letters Vol.8, pp.181-182.

Goodwin, G.C., Stefan F. Graebe and W.S. Levine (1993). Internal model control of linear systems with saturating actuators. Proceedings of the European Control Conference, 1993.

Grimble, Michael J. (2004). GMV control of nonlinear multivariable systems. Proceedings of the UKACC Control Conference, Bath 2004.

Hanus, R., M. Kinnaert and J.L. Henrotte (1987). Conditioning technique, a general anti-windup and bumpless transfer method. Automatica Vol.23, pp.729-739.

Huang, B. and S.L. Shah (1999). Performance assessment of control loops: theory and applications. Springer Verlag. Berlin.

Levine, W.S. (1996). The Control Handbook, chapter 20.1. 1st ed.. CRC Press, IEEE Press.

Majecki, P. and M.J. Grimble (2004). Controller performance design and assessment using nonlinear generalized minimum variance benchmark: Scalar case. Proceedings of the UKACC Control Conference, Bath 2004.
Seron, Maria M., Stefan F. Graebe and Graham C. Goodwin (1994). All stabilizing controllers, feedback linearization and antiwindup: A unified review. Proceedings of the American Control Conference, 1994. 